\title{
Investigation of the Electronic Structure of CdS Nanoparticles with Sum Frequency Generation and Photoluminescence Spectroscopy
}

Jingjing Wang ${ }^{1}$, Xuejiao Wu ${ }^{a, 1}$, Yuhan He, Wei Guo, Qinghong Zhang, Ye Wang ${ }^{a}$, Zhaohui Wang *

State Key Laboratory of Physical Chemistry of Solid Surfaces, The MOE Key

Laboratory of Spectrochemical Analysis and Instrumentation, ${ }^{\mathrm{a} C o l l a b o r a t i v e}$ Innovation Center of Chemistry for Energy Materials, and Department of Chemistry, College of Chemistry and Chemical Engineering, Xiamen University, Xiamen 361005, China.

AUTHOR INFORMATION

\section{Corresponding Author}

* zhwang@xmu.edu.cn. 
Table of Contents

Characterization of CdS NPs

Transient absorption measurements.

19

Setup and experiments of broadband sum frequency generation and two-photon

luminescence.

21

Figure S1 (a) XRD patterns, and (b) Steady-state photoluminescence of the CdS nanoparticles with $400 \mathrm{~nm}-600 \mathrm{~nm}$ excitation.

Figure S2 Absorption and transient absorption spectra of CdS NPs at 0 ps time resolved $528 \mathrm{~nm}$ and $590 \mathrm{~nm}$ photoluminescence decay curves excited by 377.6 $\mathrm{nm}$

Figure S4 SFG and two-photon luminescence (TPL) spectra of the hexagonal CdS NPs with visible at (a) $580 \mathrm{~nm}$, and (b) $650 \mathrm{~nm}$ : the TPL (black), background (red), raw spectrum for SFG measurement (green), and SFG spectrum (blue). 
Characterization of CdS NPs. The CdS NPs crystal structure were characterized by X-ray diffraction (Rigaku Ultima IV XRD) with $\mathrm{Cu}-\mathrm{K}_{\alpha}$ radiation and operating at 35 $\mathrm{kV}$ and $15 \mathrm{~mA}$. The scan rate was $10^{\circ} / \mathrm{min}$ in the $10^{\circ}-90^{\circ}$ region (Figure 1a). Peaks are found at $24.92^{\circ}, 26.54^{\circ}, 27.67^{\circ}, 43.68^{\circ}, 47.94^{\circ}$ and $52.1^{\circ}$, corresponding to (100), (002), (101), (110), (103) and (112) diffraction planes of the hexagonal wurtzite structure (JCPDS card No. 80-0006). The average crystallite size of the CdS NPs were calculated from the full width at half maximum (FWHM) using the Debye-Scherer formula ${ }^{1}: \mathrm{D}=\frac{0.9 \lambda}{\beta \cos \theta}$, where $\mathrm{D}$ is the crystallite size, $\lambda$ is the $\mathrm{X}$-ray wavelength of $\mathrm{Cu}-\mathrm{K}_{\alpha}$ radiation $(0.15406 \mathrm{~nm}), \beta$ is the full width at half maximum in radians and $\theta$ is the Bragg's angle. The average crystallite size of the hexagonal $\mathrm{CdS}$ NPs is about $20-40 \mathrm{~nm}$.

Optical diffuse reflectance measurements were performed using a Cary 5000 diffuse reflectance UV-vis spectroscopy (Varian) operating in the region 300-800 $\mathrm{nm}$ region at room temperature. $\mathrm{BaSO}_{4}$ was used as the reference of $100 \%$ reflectance for all measurements. Steady-state photoluminescence (PL) spectra (Figure S1b) and fluorescence excitation emission matrix spectra (EEMs) were collected using Hitachi F7000 fluorescence spectrometer (Japan). EEMs scanned in the $450 \mathrm{~nm}$ to $800 \mathrm{~nm}$ emission regime (Em) with $2 \mathrm{~nm}$ increment and with the excitation (Ex) from $450 \mathrm{~nm}$ to $600 \mathrm{~nm}$ with a $5 \mathrm{~nm}$ step. The scan speed was $2400 \mathrm{~nm} / \mathrm{min}$ and the $\mathrm{Ex} / \mathrm{Em}$ slit widths were both $10 \mathrm{~nm}$. In addition, steady state emission of CdS NPs excited at 425 $\mathrm{nm}$ by a Xe lamp were recorded using FLS980 Fluorescence spectrometer (Edinburgh). Time-resolved photoluminescence decay measurements of $528 \mathrm{~nm}$ and 
590 emissions were carried out using a time-correlated single-photon counting (TSCPC) spectrometer (Edinburgh, FLS980). Nanosecond flashlamp (nF920) was used as the excitation source $(377.6 \mathrm{~nm})$, and a photomultiplier was used as the detector. Time-resolved photoluminescence decay profiles were analyzed by nonlinear least-squares iteration procedures using FLS 980 decay analysis software with equtation1. The average lifetime $\langle\boldsymbol{\tau}\rangle$ was calculated by equation 2 .

Fit $=A_{1}+B_{1} e^{-\frac{t}{\tau_{1}}}+B_{2} e^{-\frac{t}{\tau_{2}}}$

eq.1

$\langle\boldsymbol{\tau}\rangle=\frac{\mathrm{f}_{1} \tau_{1}{ }^{2}+\mathrm{f}_{2} \tau_{2}{ }^{2}}{\mathrm{f}_{1} \tau_{1}+\mathrm{f}_{2} \tau_{2}}$ eq. 2

Transient absorption measurements. Femtosecond TA measurements were carried out with a fs Ti:sapphire laser system $(800 \mathrm{~nm}, 100 \mathrm{fs}, 1 \mathrm{kHz})^{2}$. Part of the $800 \mathrm{~nm}$ fundamental output was split into two beams. One beam focused on a $2 \mathrm{~mm}$ sapphire window to generate a super continuum white light (WLC) in $430 \mathrm{~nm}$ to $800 \mathrm{~nm}$ as the probe. Another beam focused on a BBO crystal to produce a $400 \mathrm{~nm}$ beam as the pump. The pump was chopped with a mechanical chopper running at $500 \mathrm{~Hz}$ synchronized with the laser to improve the signal-to-noise ratio. The WLC overlapped on the sample with and without the pump were separated by a galvanometer and recorded by a spectrograph (PI Acton SP2500, Princeton Instruments) with CCD camera (PIXIS 1024×1024, Princeton Instruments) after dispersion. The diameters of the pump and probe are $470 \mu \mathrm{m}$ and $160 \mu \mathrm{m}$ at the sample, respectively. The pump power was $2 \mathrm{~mW}$. The sample for TA measurement were prepared by spin-coating CdS NPs dispersed in $\mathrm{CH}_{3} \mathrm{OH} / \mathrm{H}_{2} \mathrm{O}$ solution on ITO substrates $\left(1.0 \times 1.0 \mathrm{~cm}^{2}\right)$ and drying in the dark overnight. 


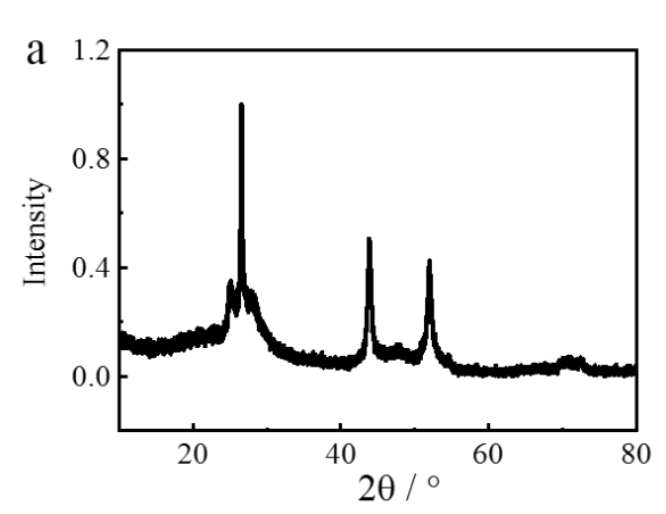

The acquisition time was also $60 \mathrm{~s}$.

\section{Setup and experiments of broadband sum frequency generation and two-photon}

luminescence. The fs Ti:sapphire laser system (Legend Elite-Duo-FS, Coherent) was described previously $y^{3-5}$. The SFG measurements were performed with a fs IR pulse ( 8 $\left.\mathrm{mW}, 150 \mathrm{fs}, \mathrm{FWHM} \sim 160 \mathrm{~cm}^{-1}\right)$ and a ps visible pulse $(4 \mathrm{~mW}, \sim 2.5 \mathrm{ps}, \mathrm{FWHM} \sim 7$ $\left.\mathrm{cm}^{-1}\right)$. The laser operated at a repetition rate of $1 \mathrm{kHz}$. The diameter of IR and visible pulse is $350 \mu \mathrm{m}$ and $390 \mu \mathrm{m}$ at the sample. The IR and visible beams were incident on the sample at a $60^{\circ}$ angle. The SFG signal was collected and recorded by a spectrograph (Andor technolopy, SR-303i-B) with an Andor CCD camera (DU920P-BR-DD). All spectra were recorded with $60 \mathrm{~s}$ acquisition time and PPP polarization combination. The two photo luminescence spectra were measured with this BB-SFG setup as well. (Only the ps visible pulses were focused on to the sample.

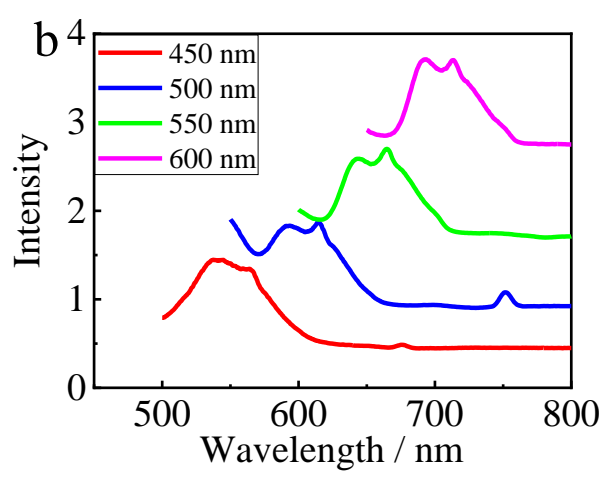

87 Figure S1 (a) XRD patterns, and (b) Steady-state photoluminescence of the CdS nanoparticles with $400-600 \mathrm{~nm}$ excitation. 

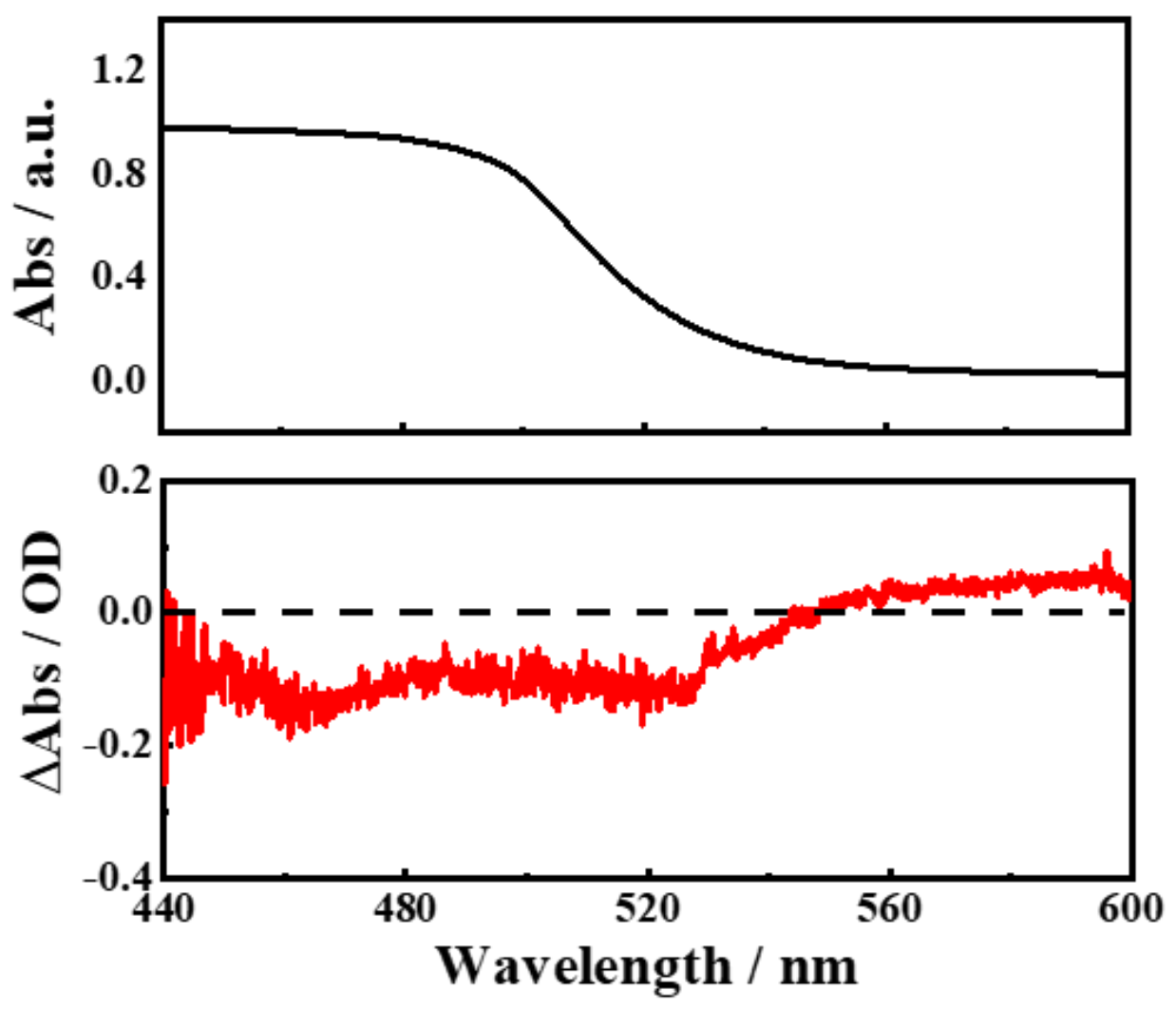

90 Figure S2 Absorption and transient absorption spectra of CdS NPs at 0 ps

91 Figure $\mathrm{S} 2$ is the absorption and TA spectrum at 0 ps. The dominant ground state

92 bleach at $465 \mathrm{~nm}$ and $528 \mathrm{~nm}$ can be assigned to the state filling of CB electronic 93 level of CdS NPs ${ }^{6}$. The redshift of the $528 \mathrm{~nm}$ bleaching from the absorption edge 94 may due to the shielded Coulomb interaction ${ }^{7}$ or the Band Gap Renormalization ${ }^{8}$ 95 caused by the photogenerated carriers. 


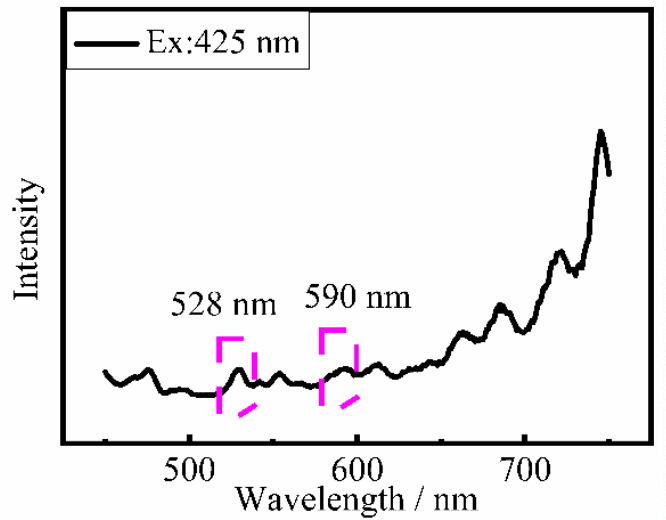

96 111 the long wavelength emission, which most likely could be the trap state 112 recombination ${ }^{12}$.

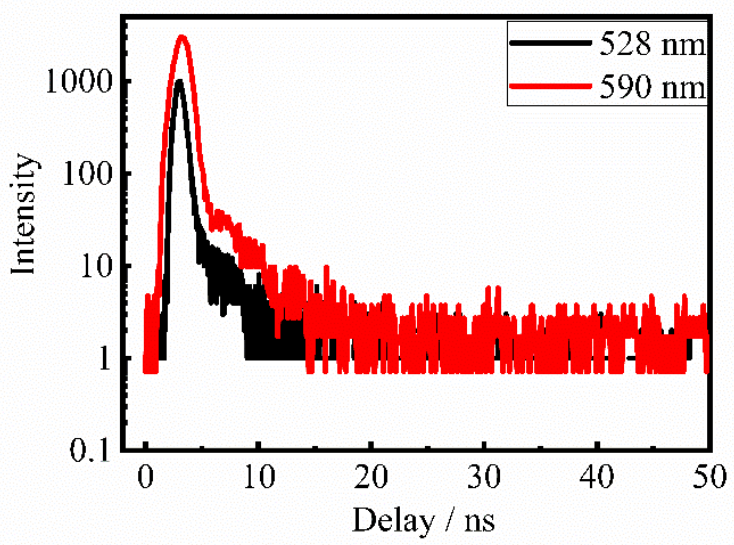

Figure S3 (a) Steady state emission spectrum of CdS NPs excited by $425 \mathrm{~nm}$ and (b) time resolved $528 \mathrm{~nm}$ and $590 \mathrm{~nm}$ photoluminescence decay curves excited by 377.6 $\mathrm{nm}$

The excitation wavelengths for the steady state emission and time-resolved photoluminescence are $425 \mathrm{~nm}$ and $377.6 \mathrm{~nm}$, which are from a Xe lamp and a Nanosecond flashlamp (nF920), respectively. Figure S3a displayed the PL spectrum, and Figure S3b displayed the decay curves of the $528 \mathrm{~nm}$ and $590 \mathrm{~nm}$ emissions. The fitting results and the average lifetimes $\langle\boldsymbol{\tau}\rangle$ are summarized in Table S1. For the 528 $\mathrm{nm}$ emission, the PL decay curve can be best fitted bi-exponentially. The $0.3 \mathrm{~ns}$ fast component can be assigned to the trapping of carriers in the surface states, that is, the decay from the band edge to the surface states ${ }^{9}$. And the $3.1 \mathrm{~ns}$ slow component is the e-h recombination ${ }^{10-11}$. The slow component is the major contribution (89.4\%). For the $590 \mathrm{~nm}$ emission, a single exponential with $3.3 \mathrm{~ns}$ time constant rather than bi-exponential can fit the decay curve very well. There is only e-h recombination in 


\begin{tabular}{llllll}
\hline & $\boldsymbol{\tau}_{\mathbf{1}} / \mathbf{n s}$ & $\boldsymbol{\tau}_{\mathbf{2}} / \mathbf{n s}$ & $\mathbf{f}_{1}$ & $\mathbf{f}_{2}$ & $\boldsymbol{\tau} / \mathbf{n s}$ \\
\hline $528 \mathrm{~nm}$ & 0.3 & 3.1 & $10.6 \%$ & $89.4 \%$ & 3.1 \\
$590 \mathrm{~nm}$ & $/$ & 3.3 & $/$ & $100 \%$ & 3.3 \\
\hline
\end{tabular}
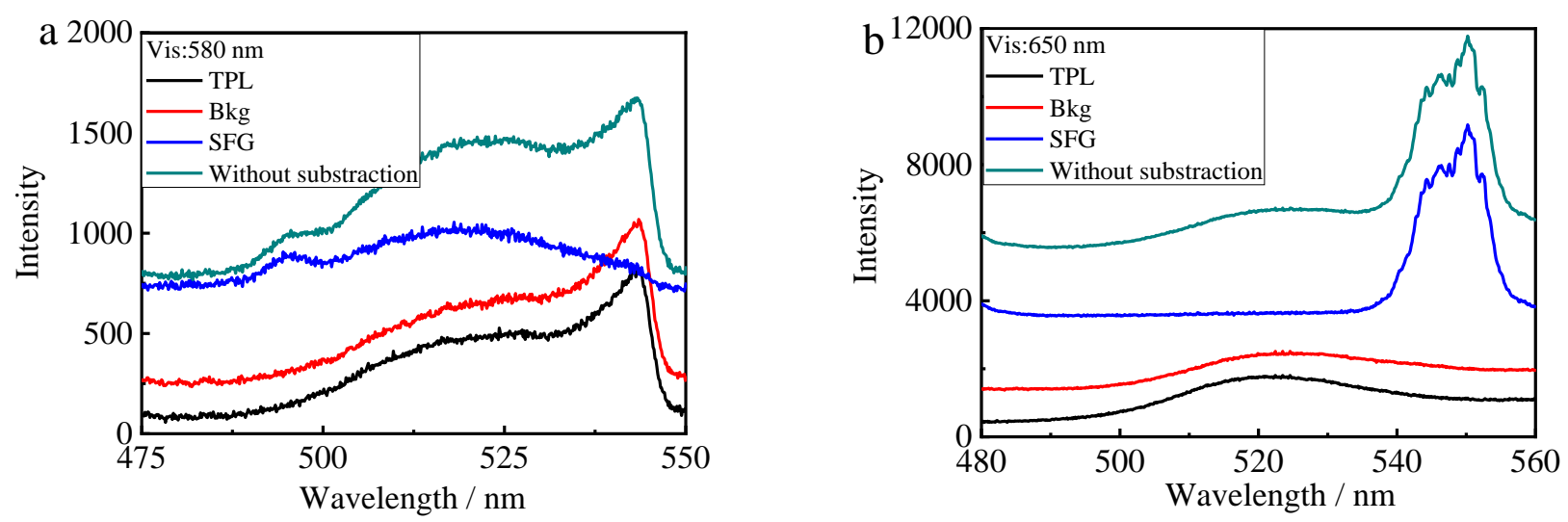

115 Figure S4 SFG and two-photon luminescence (TPL) spectra of the hexagonal CdS

116 NPs with visible at (a) $580 \mathrm{~nm}$, and (b) $650 \mathrm{~nm}$ : the TPL (black), background (red),

117 raw spectrum for SFG measurement (green), and SFG spectrum (blue).

118 The data processing procedure for the SFG spectra presented in this paper is as the

119 following: 1. Record an raw spectrum with both the IR and VIS focused on the

120 sample at certain time delay between the IR and VIS; (as the green spectra shown in

121 Figure $\mathrm{S} 4, \mathrm{IR}=3450 \mathrm{~nm}$ and VIS=580 or $650 \mathrm{~nm}$ ); 2. Record the background spectrum

122 (bkg), with both IR and VIS on the sample at a negative time delay (the VIS reaches

123 the sample before the IR) where no SFG signal generated, as the red spectra in Figure

124 S4; 3. Subtracting the bkg from the raw spectrum. In Figure S4, the blue spectra are

125 the results from this treatment. As discussed in this paper, when only the ps VIS

126 focused on the sample, TPL can be observed, the black spectra in Figure S4, which is 
127 the same as the bkg spectra (red). After the subtraction, there is still a broad spectral 128 feature around $522 \mathrm{~nm}$ in the blue spectrum of Figure S4a with VIS=580 nm, not for 129 VIS $=650 \mathrm{~nm}$. This broad feature locates at the same spectral range and with the same 130 FWHM as the TPL, which is the SFG induced photoluminescence. 


\section{References}

1. Mishra, S. K.; Srivastava, R. K.; Prakash, S. G.; Yadav, R. S.; Panday, A. C. Structural, photoconductivity and photoluminescence characterization of cadmium sulfide quantum dots prepared by a co-precipitation method. Electron. Mater. Lett. 2011, 7, 31-38.

2. Wu, X. J.; Xie, S. J.; Liu, C. X.; Zhou, C.; Lin, J. C.; Kang, J. C.; Zhang, Q. H.; Wang, Z. H.; Wang, Y. Ligand-controlled photocatalysis of CdS quantum dots for lignin valorization under visible light. ACS Catal. 2019, 9, 8443-8451.

3. He, Y.; Chen, G.; Xu, M.; Liu, Y.; Wang, Z. Vibrational dephasing of self-assembling monolayer on gold surface. J. Lumin. 2014, 152, 244-246.

4. Wang, J.; Xu, M.; Huangfu, Z.; Wang, Y.; He, Y.; Guo, W.; Wang, Z. Observation of gold electrode surface response to the adsorption and oxidation of thiocyanate in acidic electrolyte with broadband sum-frequency generation spectroscopy. Vib. Spectrosc 2016, 85, 122-127.

5. Cen, Q.; He, Y.; Xu, M.; Wang, J.; Wang, Z. Wavelength dependent resonance raman band intensity of broadband stimulated raman spectroscopy of malachite green in ethanol. J. Chem. Phys. 2015, 142, 114201.

6. Wu, K.; Du, Y.; Tang, H.; Chen, Z.; Lian, T. Efficient extraction of trapped holes from colloidal cds nanorods. J. Am. Chem. Soc 2015, 137, 10224-10230.

7. Bechstedt, F.; Glutsch, S. Nonperturbative treatment of excitons in semiconductors coherently pumped near the absorption-edge. Phys. Rev. B 1991, 44, 3638-3649.

8. Pogna, E. A. A.; Marsili, M.; De Fazio, D.; Dal Conte, S.; Manzoni, C.; Sangalli, D.; Yoon, D.; Lombardo, A.; Ferrari, A. C.; Marini, A., et al. Photo-induced bandgap renormalization governs the ultrafast response of single-layer $\operatorname{mos}_{2}$. Acs Nano 2016, 10, 1182-1188.

9. Sadhu, S.; Chowdhury, P. S.; Patra, A. Synthesis and time-resolved photoluminescence spectroscopy of capped cds nanocrystals. J. Lumin. 2008, 128, 1235-1240.

10. Sadhu, S.; Chowdhury, P. S.; Patra, A. Understanding the role of particle size on photophysical properties of cds : $\mathrm{Eu}^{3+}$ nanocrystals. J. Lumin. 2007, 126, 387-392. 11. Javier, A.; Magana, D.; Jennings, T.; Strouse, G. F. Nanosecond exciton recombination dynamics in colloidal cdse quantum dots under ambient conditions. Appl. Phys. Lett. 2003, 83, 1423-1425.

12. Lu, W.; Tokuhiro, Y.; Urnezu, I.; Sugimura, A.; Nagasaki, Y. Trap state emission of water-soluble cds nanocrystals. In Physica status solidi $c$-current topics in solid state physics, vol 6, no 1, Itoh, T.; Tanaka, K.; Schreiber, M., Eds. 2009; Vol. 6, pp 346-349. 